\title{
A PSORÍASE NO CONTEXTO DA ATENÇÃO BÁSICA: UM PLANO DE INTERVENÇÃO ATRAVÉS DE UM RELATO DE CASO
}

\author{
PSORIASIS IN THE CONTEXT OF BASIC ATTENTION: AN \\ INTERVENTION PLAN THROUGH A CASE REPORT
}

\section{6ि ACESSO LIVRE}

Citação: Santos Filho LA, Rezende MWM, Santos MAR, Miranda GP, Soares PPD, Marrafon AC, Uzan VRM, Magalhães Filho AS, Carvalho AAB (2018) A psoríase no contexto da atenção básica: um plano de intervenção através de um relato de caso. Revista de Patologia do Tocantins, 5(4): 39-43.

Instituição: ${ }^{1}$ Interno de medicina da FAPAC/ITPAC Porto Nacional-TO, Brasil. Bolsista do Programa de Bolsas de Iniciação Científica (ProBIC). ${ }^{2}$ Internos de medicina da FAPAC/ITPAC Porto NacionalTO, Brasil. ${ }^{3}$ Acadêmico de medicina da UniEvangélica, Anápolis-GO, Brasil. ${ }^{4}$ Médico. Graduado pela FAPAC/ITPAC Porto Nacional-TO, Brasil. ${ }^{5}$ Farmacêutica. Mestre em ciências da saúde pelo Hospital de Câncer de Barretos-SP, Brasil. Docente da FAPAC/ITPAC Porto Nacional-TO, Brasil. ${ }^{6}$ Médico. Mestre em Tecnologia Nuclear pela Universidade de São Paulo, Brasil. Docente da FAPAC/ITPAC Porto NacionalTO, Brasil. ${ }^{7}$ Médico. Mestre em Patologia pela Universidade de São Paulo Faculdade de Medicina de Ribeirão Preto, Brasil. Docente da FAPAC/ITPAC Porto Nacional-TO, Brasil.

Autor correspondente: Luciano Almeida dos Santos Filho ;

luciano.filho10@hotmail.com

Editor: Guedes V. R. Medicina, Universidade Federal do Tocantins, Brasil.

Publicado: 09 de dezembro de 2018.

Direitos Autorais: (c) 2018 Santos Filho et al. Este é um artigo de acesso aberto que permite o uso, a distribuição e a reprodução sem restrições em qualquer meio, desde que o autor original e a fonte sejam creditados.

Conflito de interesses: os autores declararam que não existem conflitos de interesses.
Luciano Almeida dos Santos Filho', Mateus Wendell de Moraes Rezende ${ }^{2}$, Marco Aurelio Rodrigues dos Santos ${ }^{2}$, Gabriel Pimentel de Miranda ${ }^{2}$, Pedro Paulo Dias Soares ${ }^{3}$, Augusto Cesar Marrafon ${ }^{4}$, Vanessa Regina Maciel Uzan ${ }^{5}$, Astério Souza Magalhães Filho ${ }^{6}$, Arthur Alves Borges de Carvalho ${ }^{7}$.

\section{RESUMO}

Introdução: A psoríase caracteriza-se por ser uma doença de etiologia desconhecida, com evolução crônica, acentuada e tendências às recidivas. A lesão característica é representada por uma placa eritemato-escamosa, saliente em relação a superfície da pele. Objetivos: Identificar as principais necessidades de educação em saúde de um paciente acometido por psoríase através de um relato de caso vivenciado em uma unidade básica de saúde e no próprio domicílio do paciente. Material e Métodos: A coleta de dados foi realizada através de um formulário com questões abertas e fechadas. Resultados: As principais metas para o paciente foram alcançadas por incluir melhor compreensão sobre a psoríase e o regime e adesão do tratamento, a obtenção da pele mais lisa com o controle das lesões e o desenvolvimento da auto-aceitação que é um enfretamento de estresse para realização das atividades normais e as interações sociais. Conclusões: Constatou-se que o paciente acometido com psoríase precisa está informado de sua patologia, como também toda a população que discrimina esses indivíduos. Portanto, acreditamos na educação em saúde como meio de transformação desta realidade que precisa ser mudada.

Palavras-chave: Psoríase. Educação em saúde. Qualidade de vida.

\begin{abstract}
Introduction: Psoriasis is characterized by being a disease of unknown etiology, with a chronic, marked evolution and tendencies to relapse. The characteristic lesion is represented by an erythematous-squamous plaque, protruding from the surface of the skin. Objectives: To identify the main health education needs of a patient affected by psoriasis through a case report in a primary healthcare unit and in the patient's own home. Material and Methods: The data collection was done through a form with open and closed questions. Results: The main goals for the patient were achieved by including a better understanding of psoriasis and treatment regimen and adherence, obtaining smoother skin with lesion control, and developing self-acceptance that is a stress coping for performance of normal activities and social interactions. Conclusions: It was verified that the patient with psoriasis is aware of his pathology, as well as the entire population that discriminates these individuals. Therefore, we believe in health education as a means of transforming this reality that needs to be changed.
\end{abstract}

Keywords: Psoriasis. Health education. Life quality. 
INTRODUÇÃO

Entende-se por educação em saúde quaisquer combinações de experiências de aprendizagem delineadas com vistas a facilitar ações voluntárias conducentes à saúde ${ }^{1}$.

A psoríase caracteriza-se por ser uma doença de etiologia desconhecida, com evolução crônica, acentuada e tendências às recidivas. A lesão característica é representada por uma placa eritemato-escamosa, saliente em relação a superfície da pele².

$\mathrm{O}$ adoecer traz consigo agravos múltiplos de âmbito físico, psicológico e social. O ser adoecido sofre passivamente a dupla frustração de estar doente e ter invadido e modificado toda a sua rotina de viver, muitas vezes com interferências no trabalho, em casa e em seus grupos sociais secundários.

Percebe-se que quando orientado sobre a doença e tratamento, aceita-se melhor as limitações que o agravo à saúde pode proporcionar. Muitos casos de negação da doença e não adesões ao tratamento são originadas na essência do desconhecimento do caso e/ou medo do desconhecido.

De acordo com os resultados de uma pesquisa realizada nos Estados Unidos envolvendo aproximadamente 18.000 membros da National Psoriasis Foundation, a percepção dos portadores de psoríase é a de que os médicos, na maioria das vezes subestimam o impacto da doença na vida dos pacientes, e, muitas vezes, o que é percebido pelo psoriásico como grave não é assim considerado pelo médico ${ }^{3}$.

Como acadêmicos de medicina, voltar-se-á atenção ao enfoque educativo ao ser abordado por uma pessoa da comunidade rural que relatou incômodo por sentir-se rejeitado socialmente por portar uma patologia pouco conhecida por ele próprio. Este fato levou a estudar cada vez mais o assunto para responder a esta pessoa suas principais interrogações ao passo que pretendia obter sua adesão ao tratamento.

Nossa proposta tem procurado lidar com a complexidade e os desafios postos pela educação em saúde na área da formação profissional, orientando-se para o desenvolvimento de intervenções educativas que apontem para a reflexão contínua e ressignificação das ações cotidianas, como também para a integração das perspectivas técnica, cognitiva, afetiva e político-social, mediante a uma compreensão multidimensional do processo educativo ${ }^{4}$.

Educação em saúde é reflexão-ação fundada em saberes técnico-científicos e populares, culturalmente significativos, exercício de espaço democrático, capaz de provocar mudanças individuais e prontidão para atuar em grupo, interferindo no controle e na implementação das políticas públicas, contribuindo para a transformação social ${ }^{5}$.

Neste sentido, este estudo objetiva identificar as principais necessidades de educação em saúde de um paciente acometido por psoríase através de um relato de caso vivenciado em uma unidade básica de saúde e no próprio domicílio do paciente. Pretende-se contribuir ainda com a construção do conhecimento no sentido de elucidar o comportamento clínico e educativo nesta população para subsidiar medidas de controle, além de incitar a realização novos estudos sobre a temática.
Para a realização do caso foi utilizada a pesquisa descritiva com uma abordagem qualitativa. O estudo foi realizado em uma unidade básica de saúde, na zona rural, em um município do Estado do Ceará, visto em momento oportuno e de experiência em prática enquanto acadêmicos de medicina a um paciente acometido por psoríase que necessitava de uma intervenção educativa e clínica. Após identificação e interesse pelo caso decidimos fazer o acompanhamento no domicílio através de visitas domiciliárias.

O sujeito do estudo foi composto por um paciente com psoríase a qual buscou informações para tirar suas dúvidas juntamente ao profissional médico da unidade de saúde e acadêmicos de medicina, os mesmos procuraram intervir através de dados clínicos e educação em saúde. Constitui-se de um paciente de 63 anos do sexo masculino, casado. A seleção do sujeito da pesquisa ocorreu de forma aleatória, posto que o próprio paciente buscou informações sobre sua patologia junto ao serviço de saúde e profissionais.

Para a coleta de dados foi utilizada a técnica de entrevista semiestruturada, composta por questões abertas e fechadas, orientada por um formulário único, contendo dados relativos à patologia em questão, dados de identificação, antecedentes pessoais e familiares, percepção e expectativas, educação em saúde, atendimento das necessidades básicas e exame físico.

Ao todo foram realizadas três visitas domiliárias nos meses de junho e julho de 2017. Sendo posteriormente realizadas através da identificação dos problemas e planejadas as ações e intervenções. Cada visita realizada teve duração mínima de quarenta minutos.

Os acadêmicos de medicina participaram do estudo por o paciente está ciente das informações prestadas para auxiliar no seu quadro clínico, assegurando privacidade e integridade da vida pessoal. O paciente assinou um termo de consentimento informado de caráter livre esclarecido, onde em todas as suas etapas respeitou os princípios contidos na Resolução no 466/2012 do Conselho Nacional de Saúde/ Ministério da Saúde, que dispõe sobre as pesquisas envolvendo seres humanos ${ }^{6}$.

\section{DESCRIÇÃO DO CASO}

Paciente de 63 anos, sexo masculino, casado, evangélico, procedente da zona rural do Estado do Ceará, motorista, possui ensino fundamental completo, com residência fixa e renda individual de três salários mínimo e renda familiar de quatro salários mínimo. Sua moradia possui seis cômodos, acesso a transportes, possui rede de esgoto tipo fossa, iluminação, abastecimento de água e coleta de lixo. Moram três pessoas e ainda cria-se animais domésticos como gato. Informa ter vacinação incompleta e ausência de patologias como: câncer, diabetes, AVC, hemofilia, cardiopatia e nunca ter feito hemotransfusão. Já foi internado uma vez por ter submetido a uma cirurgia de cisto na região cervical. Tabagista e etilista há mais ou menos 45 anos. Possui história familiar de hipertensão (mãe falecida), pai e irmão terem falecido de câncer. Possui doença crônica como psoríase e hipertensão arterial. Faz uso de captopril e nada atualmente para psoríase. Diz ter deixado de fumar e ingerir bebida alcoólica há 5 anos. Relata não praticar nenhuma atividade 
física. Informa está sentindo-se bem no momento. Refere ter começado o quadro de psoríase há 18 anos. Diz ter procurado serviço médico para tratamento das lesões apresentando inicialmente nos joelhos e cotovelos. Em várias consultas médicas relata tratar de uma simples micose que logo desaparecia, fazendo com que o paciente desacreditasse e automedicar-se. Tem como lazer: conversar com os amigos, assistir jogos de futebol e brincar com os netos. Refere dormir bem. Alimenta-se quatro vezes por dia, come alimentos bem diversificados e mantém apetite conservado após a doença. Bebe pouca água, aproximadamente um litro por dia. Tem eliminações intestinais sem queixas, uma vez por dia, de consistência, cor e odor normais. Têm micção sem queixas, quantidade e frequência normal. Apresenta boa higiene corporal, sendo independente para realizá-la. Toma banho duas vezes por dia, realiza escovação dos dentes duas vezes por dia. Corta as unhas, cabelos e barba quando crescem. Diz não ter vida sexual ativa. Relata ter realizado exame de detecção de câncer da próstata e apresenta queixas quanto a sua sexualidade. Diz não saber bem o seu problema, pois gostaria de estar informado sobre tratamento, alimentação e quadro clínico da psoríase. Comenta ainda não está informado de qual serviço deveria buscar para tratamento das lesões, devido dúvidas de orientações e prescrição de medicamentos, sem diminuição das lesões.

\section{EXAME FÍSICO}

Paciente higienizado, com expressão facial normal, sem sinais de desconforto ou dor, deambulando independentemente. Vestuário limpo. Orientado, consciente, verbaliza diálogo com ordenação lógica do pensamento. Medidas antropométricas: peso $-87,0 \mathrm{Kg}$, altura $-1,72 \mathrm{~cm}$. Sinais vitais: PA- $140 \times 90 \mathrm{mmHg}, \mathrm{T}^{\circ}-36^{\circ} \mathrm{C}$, pulso- $62 \mathrm{bpm}$, respiração- 17 irpm. Ao exame físico da cabeça apresenta couro cabeludo sem sujidade ou parasitose e com lesões psoriásicas. Crânio sem anormalidades. Face simétrica, coloração uniforme. Estruturas externas dos olhos e ouvidos sem anormalidades. Ouvido íntegro, indolor e sem sujidade. Possui boa acuidade visual e auditiva. Mucosa nasal sem lesões, secreções e sangramentos. Lábios íntegros e úmidos, mandíbulas e glândulas parótidas sem anormalidades. Mucosa oral íntegra de cor rosada e aparência normal. Faz uso de prótese dentária com higiene oral adequada. Inspeção e palpação de pescoço sem presença de anormalidades. Alinhamento da traqueia centralizado, ausência de aumento ou diminuição da glândula tireoide. Nódulos linfáticos sem tamanho alterado. Presença de pulsos carotídeos com frequência, ritmo e amplitude uniformes. Artéria jugular sem distensão aumentada de pulsação. Tórax com presença de lesões psoriásica na região inframamilar, com simetria, coloração hiperemiada e forma irregular. Apresenta tipo respiratório torácico, ritmo respiratório normal. Avaliação cardíaca com inspeção, percussão, palpação e ausculta sem anormalidades. Verificação de pulso apical, normoesfígmico e sem arritmias. Mamas e axilas sem alterações e linfonodos sem alteração de tamanho. Presença de nevos marromacastanhados espalhados na região do tórax e psoríase gutata. Abdome globoso, com peristaltismo presente, indolor a palpação. Membros superiores simétricos e sem deformidades, rede venosa visível, presença de lesões psoriásicas na região dos cotovelos com bordas eritematosas e escamosas. Unhas com sinais de fungo com comprometimento no leito ungueal e coloração atípica. Membros inferiores simétricos e com lesões psoriásicas nos joelhos. Unhas com sinais de fungo com comprometimento do leito ungueal. Diz nádegas com presença de lesões psoriásicas. Genitais e ânus com ausência de secreções atípicas.

Apresentamos aqui as três evoluções que ocorreram dentro da fase de orientações e avaliações das visitas domiciliárias.

\section{1a visita domiciliária realizada em 05 de junho de 2017}

Paciente de 63 anos, sexo masculino, com diagnóstico inicial de psoríase gutata, inspira cuidados. Consciente, orientado, com estado emocional triste. Nível de higiene e arrumação adequada. Deambulando independentemente. Dieta inadequada de acordo com os padrões dietéticos. Sono e repouso tranquilo. Hemodinamicamente estável. Pele ressecada, com lesões psoriásicas de bordas eritematosas, escamosas e hiperemiadas de grande extensão nas regiões do couro cabeludo, tórax, cotovelos, nádegas e joelhos. Presença de unhas dos membros inferiores e membros superiores com lesões psoriásicas acometendo o leito ungueal. Normocorado, com boa perfusão e sem presença de edema. Eliminações urinárias e intestinais de aspecto, quantidade, frequência e odor normal. Ao índice de massa corporal resultou em 25,2 $\mathrm{Kg} / \mathrm{m} 2$, classificado como sobrepeso. Refere negligência de profissionais da saúde a qual procurou serviço para tratamento das lesões, no entanto diz não tendo resposta e eficácia da melhoria do quadro clínico resolveu desistir da terapia medicamentosa, segundo diz inadequada por as lesões está aumentando. Relata desconforto físico por as lesões acometer sua estética e discriminação de alguns membros da comunidade por pensar que as lesões são contagiosas. Diz não saber muito sobre a patologia e restrições que devem ser implementadas no seu dia-a-dia. Foram realizada entrevista, exame físico, verificação de sinais vitais. Recebe orientações para procurar centro de referência em dermatologia do Estado do Ceará, e o mesmo sendo referenciado. Realizado reforço sobre cuidados com as lesões, quadro clínico da patologia, autocuidado, compreensão sobre o regime de tratamento e alimentação.

\section{2a visita domiciliária realizada em 23 de junho de 2017}

Paciente logo no início da visita apresenta resultado de histopatológico, com hipótese diagnóstica de psoríase vulgar em placas e prescrição de metotrexato. Apresenta estado geral melhorado, consciente, orientado, humor alegre, nível de higiene e arrumação preservada. Deambula independentemente. Aceita dieta, não sendo intolerante as restrições. Sono e repouso preservado. Parâmetros vitais estáveis. Pele e mucosa hidratada, normocorada, com lesões psoriásicas no couro cabeludo, cotovelos, joelhos, nádegas e comprometimento do leito ungueal. Informa está positivo para melhora do estado clínico. Reforçado orientações sobre o tratamento e realizada nova sessão de informações sobre dieta, autocuidado das lesões e mudanças no estilo de vida.

\section{3a visita domiciliária realizada em 14 julho de 2017}


O encontro durou cerca de quarenta minutos, com início às 9h30min. Paciente encontra-se com estado geral bom, consciente, orientado, humor alegre, nível de higiene e arrumação preservada. Deambula normalmente. Aceita dieta, não sendo intolerante as restrições. Sono e repouso preservado. Hemodinamicamente estável. Apresenta pele com lesões psoriásicas com controle nas regiões acometidas. Demonstra conhecimento e compreensão do processo patológico e seu tratamento. Refere estar mais tranquilo e ciente das eventuais recidivas. Realizadas orientações sobre importância da adesão ao tratamento e acompanhamento no serviço especializado, como também cuidados específicos de lubrificação da pele. No final desta visita o paciente encontrase orientado em relatar o que é a psoríase, descreve o estresse emocional como fator deflagrador, demonstra a administração correta da medicação tópica e sistêmica e expressa otimismo sobre os resultados do tratamento. Acadêmicos de medicina acrescem comentários sobre como se sentiu na realização de cada visita e os recursos empregados como enaltecedores de conhecimentos através da aplicação das entrevistas e contexto inlocu da realidade do paciente. A prática da utilização da entrevista fundamentou o estudo, pois para iniciar esta prática necessitou do conhecimento do médico do serviço de saúde e da literatura pertinente. Diante dos desafios propostos, procuremos através do exame clínico junto ao paciente analisar as informações colhidas, daí identificarmos os problemas vigentes e potenciais. Para tanto, foi estabelecida a aplicação de intervenções que atendeu as necessidades do paciente de maneira holística e humanitária através da educação em saúde.

\section{RESULTADOS E DISCUSSÕES}

As informações e dados coletados foram consolidados e organizados após três dias de visitas domiciliárias, sendo possível observar os seguintes resultados que apontam para a dificuldade de conviver com a doença havendo a princípio revolta, assim como dificuldade de adaptação às condições sociais necessárias para estabilização do quadro e as novas condições de vida.

Foram identificadas e trabalhadas educativamente necessidades de orientação quanto à fisiopatologia e quadro clínico da psoríase, terapêutica e cuidados alimentares.

Observou-se uma dieta inadequada nos pacientes com psoríase. O desconhecimento da importância dos alimentos, de fazer refeições regulares, a facilidade de alimentos prontos à disposição, com excesso de calorias e carboidratos, e a falta de determinação para conciliar horários de trabalho e alimentação são fatores que, nestes pacientes, contribuem para a obesidade, deficiência nutricional e piora da psoríase ${ }^{7}$.

Ao entender o paciente acometido com psoríase foi visto que o estresse é um fator bastante relevante para aumento das lesões, pois o mesmo pode ser uma das causas deflagradoras.

O paciente procurou após as orientações serviço especializado de saúde para controle e adesão ao tratamento, após ser referenciado.
Ao término das visitas domiciliárias o paciente demonstrou conhecimento e compreensão do processo patológico, descrevendo a psoríase e a terapia prescrita. Dizendo manter o controle da terapia adequada, demonstrando a aplicação correta da terapia tópica. Ao avaliar os resultados houve feedback positivo ao passo que a pele apresenta-se mais suave e alcança o controle das lesões, não apresentando novas lesões, porém mantendo a pele lubrificada e macia. Ao aspecto psicológico apresentou e desenvolveu a auto-aceitação no decorrer das visitas, expressando o otimismo sobre os resultados do tratamento.

As principais metas para o paciente foram alcançadas por incluir melhor compreensão sobre a psoríase e o regime e adesão do tratamento, a obtenção da pele mais lisa com o controle das lesões e o desenvolvimento da auto-aceitação que é um enfretamento de estresse para realização das atividades normais e as interações sociais.

O sucesso no tratamento da psoríase compreende três princípios. Em primeiro lugar é essencial que este seja adequado às expectativas dos doentes, em segundo lugar deve-se maximizar os recursos existentes, ou seja, as terapêuticas convencionais. Por último, ponderar a utilização das novas terapêuticas respeitando as indicações formais existentes $^{8}$

A atuação do médico deve ser importante para orientar o paciente quanto ao tratamento, explicando-o a cronicidade da patologia e os cuidados com higiene usando emolientes para hidratar o extrato córneo e evitando a secagem das lesões. Alimentação equilibrada e estilo de vida saudáveis são fundamentais à estabilidade da doença. Estratégias de enfrentamento de estresse e o apoio familiar aumentam a autoestima do psoriásico.

A visão dos acadêmicos de medicina acerca de suas experiências neste relato demonstrou extrema confiança dos resultados esperados de suas orientações para resultados positivos que pudesse mudar o quadro clínico do paciente e auxiliar nas dúvidas que persistia da doença.

Em termos de qualidade de vida, a imagem corporal é a única variável preditiva do modelo. Quanto mais negativa a percepção da imagem corporal maior o impacto negativo na qualidade de vida. Tais resultados podem indicar que a satisfação com a imagem corporal ou não, é um indicador do bem-estar ${ }^{9}$.

\section{CONCLUSÕES}

Identificamos que a medicina tem um papel fundamental na educação em saúde, aliviando temores e elucidando dúvidas que contribuirão significativamente na adesão ao tratamento e melhoria do quadro clínico. Nesse contexto a medicina deve se inserir promovendo a humanização do cuidado dentro ou fora do serviço ambulatorial/hospitalar e orientando continuamente a comunidade sobre dermatoses, como psoríase.

Ficou evidente a necessidade de uma prática humanística e capaz de transformar estratégias em realidade para o desenvolvimento de ações enriquecedoras da medicina na vivência da comunidade e no dia a dia de cada indivíduo. No entanto, as dificuldades são muitas, mas o desejo de melhoria nos trouxe o encorajamento da busca e atenção ao 
paciente que há tempo tinha o diagnóstico da doença sem perspectiva de avanço e redução das lesões.

Constatou-se que o paciente acometido com psoríase precisa estar informado de sua patologia, como também toda a população que discrimina esses indivíduos. Portanto, acreditamos na educação em saúde como meio de transformação desta realidade que precisa ser mudada.

\section{REFERÊNCIAS}

1. Candeias NMF. Conceitos de educação e de promoção em saúde: mudanças individuais e mudanças organizacionais. Rev. Saúde Pública. v. 31, n. 2, p. 209-13, 1997.

2. Silva KS, Silva EAT. Psoríase e sua relação com aspectos psicológicos, stress e eventos da vida. Estudos de Psicologia. Campinas. v. 24, n. 2, p. 257-266, abr-jun. 2007.

3. Martins GA, Arruda L, Mugnaini ASB. Validação de questionários de avaliação da qualidade de vida em pacientes de psoríase. Anais brasileiros de dermatologia. Rio de Janeiro, v.79, n.5, p.521-535, 2004.

4. Vasconcelos EM (org.). A saúde nas palavras e nos gestos: reflexões da rede educação popular e saúde. 1a ed. São Paulo: Hucitec, 2001. Cap. 5.

5. Barroso GT. et al. Educação em saúde no contexto da promoção humana. Fortaleza: Edições Demócrito Rocha, 2003. p. 31-36.

6. Conselho Nacional de Saúde. Diretrizes e normas regulamentadoras de pesquisa envolvendo seres humanos. Resolução 466. Brasília: Ministério da Saúde, 2012.

7. Festugato M. Estudo piloto sobre alimentos que devem ser evitados por portadores de psoríase. An Bras Dermatol. 2011; 86 (6): 1103-8.

8. Pinto GM, Filipe P. Normas de boa prática para o tratamento da psoríase em placas em idade não pediátrica com biológicos. Revista da SPDV, 2011; 69 (4): 531-53.

9. Brito L, Pereira MG. Variáveis individuais e familiares na psoríase: um estudo com doentes e parceiros. Psicologia: teoria e pesquisa, 2012; 28 (2): 171-19. 